\title{
SECURING WEB SERVICES USING SEMANTIC WEB TECHNOLOGIES
}

\author{
Brian Shields, Owen Molloy, Gerard Lyons and Jim Duggan \\ Department of Information Technology, \\ National University of Ireland, \\ Galway, Ireland. \\ brian.shields@geminga.it.nuigalway.ie, \{owen.molloy,gerard.lyons,jim.duggan\}@nuigalway.ie
}

\begin{abstract}
In this paper we propose an authorisation definition and access control solution for Web Services. In our proposal we define our access control policies using an OWL-DL language based on the eXtensible Access Control Markup Language (XACML). We propose the use of resource and subject metadata ontologies, also written in OWL. We then present a complete Web Services architecture which incorporates this access control model. As part of this architecture we propose a novel document filtering mechanism according to the semantic enriched access control policies.
\end{abstract}

Keywords: Web Services Security, XACML, OWL, Semantic Web, Access Control

\section{Introduction}

The World Wide Web is growing at a exponential rate [1]. There are more and more technologies being developed to provide different ways of accessing this huge information resource, as well as representing the information stored. Because of the increase in information available and of people or agents accessing it, the issue of securing this information has become paramount.

Access control is the current 'hot topic' in information security. It has become necessary to define security policies that allow a person describe who can access what information, where they access it from, when they can access it and how they can access it. However, as each new item of information is added to this secure environment, is it necessary to define all of these policy issues again? Does each new user have to be added to all of these access lists? Unfortunately until recently the answer was yes. There have been strides taken in the advancement of security policies on the Web. Role Based Access Control has become commonplace, where a new user can be granted all the permissions of a particular role or group. 
This paper will focus on access control for the Web Services environment and how these access control practices can be improved by enriching them with machine processable semantics. Therefore not only can we define users as part of groups or roles, but we can now group stored information according to its meaning. This paper proposes a way of augmenting existing Web Service access control standards to become semantically-aware. We define a security architecture which incorporates this novel access control policy. We provide a novel document filtering algorithm according to access control policies, therefore not limiting access control to a document to a boolean value.

In the next section we discuss the two principal standards which will be used from both the Web Services security field and the Semantic Web arena. We then present our solution which includes a proposed policy language, limited document access algorithm and a security architecture which encompasses these. We have included a section on related research in this field and present our conclusions.

\section{Technologies}

It is important when developing a solution to be aware of standards and standard practices in the area. For the solution we propose in this paper we straddle two key areas of todays World Wide Web research: Web Services security and the Semantic Web. From the standards in Web Services security we focus on XACML. The Semantic Web technology used is OWL. This section introduces these standards.

\subsection{XACML}

XACML or eXtensible Access Control Markup Language is defined by the OASIS standards body [2]. XACML is an XML based language used to construct access control policies for Web Services environments. XACML can grant or refuse access to protected resources based on attributes of the requester, the protocol used to access the resource, authentication methods or even global settings such as time of day and location.

There are six principal components of the XACML architecture:

- PEP, or Policy Enforcement Point, intercepts a SOAP request and constructs a SAML authorisation decision query from the information in the request. Rules pertaining to the access query, while they may be defined and evaluated elsewhere, are enforced here.

- PDP, or Policy Decision Point, evaluates the rule or rules in the policy. The policy can be retrieved from the PRP if it is not cached on the PDP. Once the policy has been evaluated, a SAML authorisation decision assertion is returned to the PEP. 
- PRP, or Policy Retrieval Point, returns the requested policy to the PDP. If the policy is not available at the PRP it can be retrieved from the policy store. This will be the case if the policy has never been requested before, or if the policy is being refreshed.

- PIP, or Policy Information Point, is used to calculate the predicate of a rule. In XACML, the predicate is defined as "the ability to query an attribute" [2]. The attribute information is returned to the PRP, from where it was requested, in the form of a SAML attribute assertion.

- PAP, or Policy Administration Point, creates rules, combines rules into policies, and uploads these policies to the policy store. The PAP usually takes the form of a graphical console and uploads the policies as XACML.

- Policy store is used to store the rules and policies defined at the PAP. While XACML is used for the import and export of policies, they are not necessarily stored in their native format. They may be stored in a traditional relational database with an XML interface.

There are three essential parts to XACML policy writing:

- Rule: A rule is the most elementary piece of a policy. Rules are encapsulated inside a policy. Rules are evaluated based on their contents. The main components of a rule are a target, which is a set of resources, subjects or actions to which the rule is intended to apply; an effect, which contains the rule writers intended consequence if the rule is evaluated to be true; and a set of conditions.

- Policy: According to O'Neill [3], this is "perhaps the most important aspect of the specification". A policy contains a set of rules, an algorithm for combining these rules, a target, similar to that in rule, and a set of obligations. An obligation is defined as an action to be performed once the authorization decision is complete.

- PolicySet: This is a set of policy elements, a policy combining algorithm, a target and a set of obligations.

\subsection{OWL}

OWL [4] was created by the W3C Web Ontology working group. It is based on the DAML+OIL language, and is layered on top of RDF and RDFS. OWL actually consists of three sub languages or dialects: OWL Lite, OWL DL and OWL Full. These dialects form a layered pattern as seen in Figure 1.

Figure 1 shows that OWL Lite is a subset of OWL DL which is in turn a subset of OWL Full. OWL Lite is usually used to express simple classifications and relationships. OWL DL, or OWL Description Logic, contains all OWL constructs but has certain limitations necessary to guarantee computational completeness and decidability. OWL Full contains all OWL constructs. 


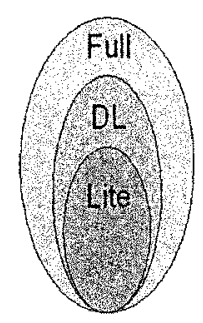

Figure 1. OWL Dialects Layering [5]

OWL Full cannot guarantee process completeness. The principal limitation of OWL DL is the restriction that classes cannot be instances. This is a necessary restriction for completeness.

An OWL ontology consists of a number of classes, properties and instances. Classes have definitions describing their characteristics. Properties have characteristics such as transitivity or functionality as well as some domain or range information. Individuals have a class membership, one or more relationships to other individuals and a concrete value.

\section{Proposed Solution}

\subsection{Framework Architecture}

Although a security solution such as this is almost completely composed of or related to access control, there are a number of other services which must be in place to offer a complete security framework. These services include a Key Management Service, an Encryption and Decryption Service and a Framework Management Service.

Figure 2 shows the proposed architecture of the proposed security framework which will be discussed in the remaining part of this section.

Key Management Service. The security framework will provide a service which will create, manage and store X.509 digital certificates. These certificates will be used as security tokens in requesting SOAP message headers to provide a non-repudiative user identity.

The Key Management Service will be designed and implemented using the XKMS (XML Key Management Specification) Standard from OASIS [6]. This provides two principle services:

- XML Key Information Service Specification (XKISS) - This service locates a public key in order to encrypt information for an individual or to verify signed information. 


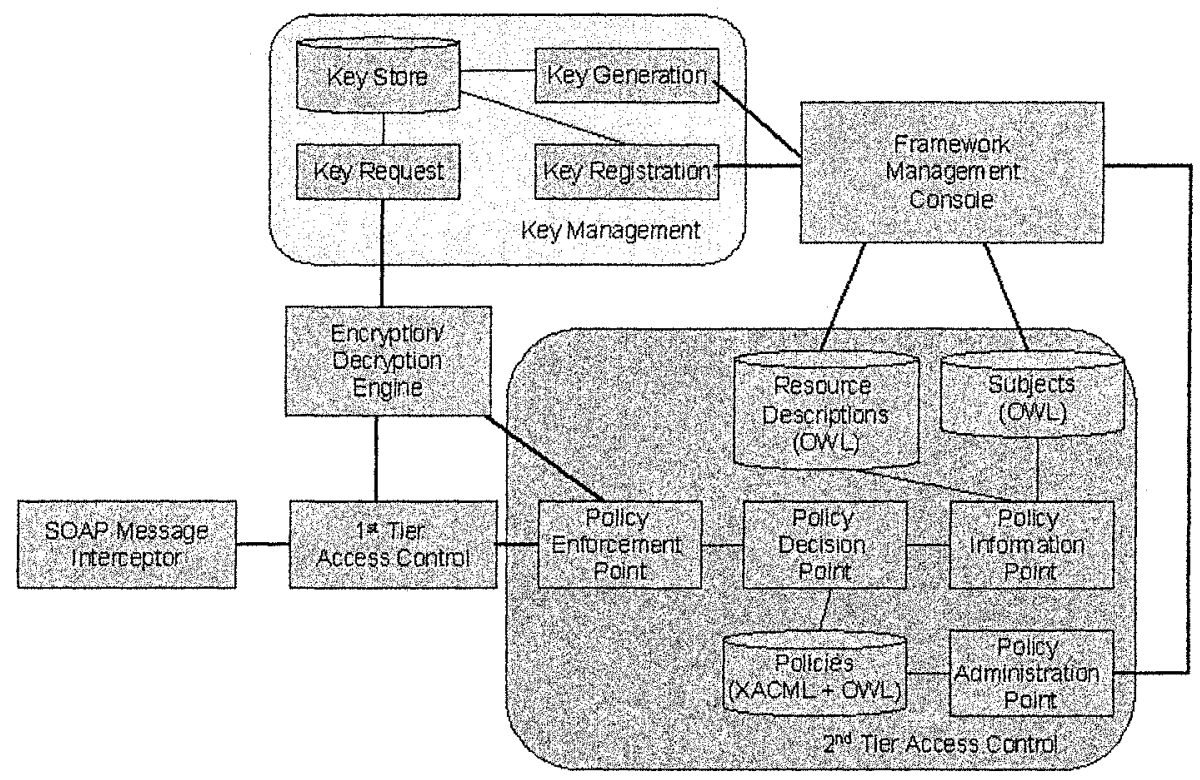

Figure 2. Proposed Security Framework Architecture

- XML Key Registration Service Specification (XKRSS) - This provides a number of services to register, recover, reissue and revoke keys.

As well as implementing the services specified in XKMS, keys will be stored locally by the security framework in order to reduce the interruption time between a user requesting a service and when that service is called. Keys for new users will be registered or created through the Framework Management Service.

Encryption and Decryption Service. As well as protecting information while in storage, the Security Framework must enforce a strict security policy on the confidentiality of information while 'on the wire'. All communication between remote clients and the Security Framework will be encrypted by the sender: be that the client or the framework. Additional encryption policies may be specified by the designers of the Web Service endpoints.

The Encryption and Decryption services will be exposed services from the encryption/decryption engine. This engine will encrypt and decrypt information according to a public key. If User_A makes a Web Service request to a Web Service managed by the Security Framework, the SOAP request is, at the very least, encrypted using our public key. The request will be subject to first 
tier authorisation (detailed in Section 3.2); upon successful authorization, the message will be fully decrypted by the encryption/decryption engine, using our private key, and passed to second tier authorization. The response returned to the requestor is encrypted using their public key which is located using the Key Management Service.

Framework Management Service. The Framework Management Service will be a HTTP and SOAP management centre used by the administrators of the Security Framework. It is essentially a front-end management of the different components of the framework. It will have five principal responsibilities:

- Uploading and registering or creating new keys

- Register valid Web Service endpoints

- Create/edit/remove access control policies

- Add/remove/edit users and semantic user descriptions

- Add/remove/edit semantic resource descriptions.

\subsection{Access Control Model}

The limiting of access to resources in this system will be done on a two tier level. The first tier

- Validates that the client requesting the resource is a registered user of the system and has a digital certificate to prove their identity

- Verifies that the requested Web Service end point exists and is registered with the security framework.

Failing either of these two checks will result in a security error and the request will not be passed to tier two.

The second tier of the access control model provides, rejects or limits access to the protected resources according to stored policies. This tier of the access control model will be similar in architectural design to that of the XACML standard. The purpose of the second tier is to define and control what the person can access; this can have three possible results:

- Access denied

- Full access granted

- Access granted but restricted.

The first two elements are straightforward. In the first case, the Web Service request is terminated and the response is returned to the user with the appropriate security exception; the second case results in the request being passed to the appropriate endpoint, free from all encryption, and the response is returned to the user that requested it. The third element is more complicated; although the request is permitted to be passed to the requested endpoint, the response 
must be examined according to the relevant policy rules to ensure illegal information access is not occurring. This scenario is common where a document of some sort is requested from an endpoint by a user that is allowed only limited access to the document. The Web Service request must be allowed to continue. However, the response must be intercepted to remove the appropriate fields. This is discussed in more detail in the following sections.

For this level of access control, it is necessary to provide a means of representing policies (access rules) which define the access rights that will be implemented in the second tier. We will continue to use the XACML standard, however augment it with semantics. Using a semantically-aware access control language increases the flexibility and power of the constructed policies. The next section defines the proposed policy language for use in this security framework; the following section presents how the subjects and resources, about which the policies are written, are described semantically. Policy evaluation is explained by describing how the policies written using the proposed language are evaluated according to the descriptions of the policy subjects and resources. The final section describes how access to documents can be limited without being fully removed.

Policy Language. The policy language for this security framework will be an OWL representation of XACML. The main expressions or constructs from XAMCL will be represented in OWL-DL atomic classes. From initial studies of both technologies the principal classes in our new language will be:

- PolicySet - This contains a set of policies; related policies will be grouped into sets.

- PolicyCombiningAlgorithm - When there is more than one policy in a policy set, there must be an algorithm to define precedence and conflict resolution.

- Policy - The policy contains a target, a set of rules and a rule-combining algorithm.

- Target - This specifies the subjects, resources, actions and environment to which the policy applies.

- Subject - This represents the subject to which the policy applies.

- Resource - This represents the resource to which the policy is protecting.

- Action - This is the resulting action which can or must take place when a policy is evaluated.

- Environment - This represents the environment attributes which must be present or absent from the request.

- RuleCombiningAlgorithm - This acts in the same way as the PolicyCombiningAlgorithm, on a rule level. 
- Rule - Each rule contains a target, a condition or set of conditions, and an action or set of actions.

- Condition - This represents one condition of a rule.

- Effect - This represents the consequence of a rule evaluated to be true.

- Obligation - This represents an action which must take place as well as enforcing the access control decision. They can be defined at a policy or policy set level and are only executed if the appropriate policy or policy set is evaluated.

An OWL reasoning engine will be constructed to evaluate the semantically aware rules against the subjects and resources which are discussed in the next section.

Policy Subjects and Resources. The subjects and resource descriptions will be OWL ontologies that can be referenced in the policy rules. The subjects and resource descriptions will be domain specific. These ontologies will be built with an external tool, Protégé [7] for example, and will be uploaded to exposed interfaces using the management framework.

Policy Evaluation. Since the policy language and information representation is constructed using OWL-DL, Racer, an existing OWL reasoning engine, will be used as the basis for the Policy Decision Point. However, it will be necessary to extend this to provide for the obligations as described earlier.

Policy evaluation will take a number of steps. When a request is passed to the tier two access control, it is parsed by the Policy Enforcement Point (PEP) which will determine what the user is requesting. The PEP will then request a policy decision from the Policy Decision Point (PDP). The PDP will determine which policies apply to this request and source them from the policy store. To evaluate the policies, the PDP will have to request subject and resource description information from the Policy Information Point (PIP). Once the policies are evaluated, the decisions are returned to the PEP for enforcement.

Limited Document Access. The security offered by our security framework will be an XML element level. This fine grained level of access control is required in many of today's security environments, as there are many people at different levels of an organisation's hierarchy with different levels of access to documents, and even within one document. The documents will be defined semantically at an element level, which will enable the element level control to be decided at the evaluation by a semantic reasoner.

Enforcing this level of control is envisaged to occur in two steps, first at the point where the Web Service request enters the system, and the second as the response is leaving. Figure 3 shows the flow of what happens in this scenario. On the receipt of the request, first-tier access control is enforced; after passing 
this tier the second-tier of access control is enforced. This will have one of the three results defined in Section 3.2. In the case of the first two results (access denied or full access granted), the request will proceed as normal. In the case where there is a document or part of document requested from the Web Service, the service will be called normally but there will be a flag set in the response interceptor to prune the document being returned in the message, according to the semantic rules triggered in the reasoning engine.

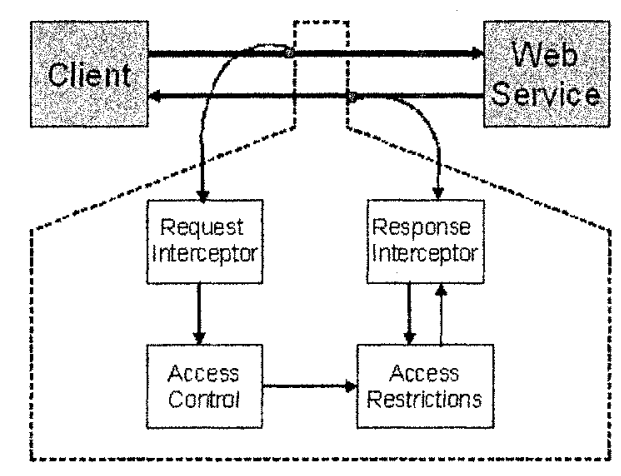

Figure 3. Limited Document Access Control Model

\section{Case Study: Health Sector}

Access control and privacy is critical in the health sector. There are innumerable documents and items of information in one active hospital. This fact, coupled by the countless levels of access by different groups, creates a monolithic task out of defining access control rights or rules. Enforcing these rules can be just as problematic. When the hospital administration eventually put a system in place, which is more often than not static, it is not portable to other hospitals in the health service. This is an activity that must be carried out at each location.

We propose the use of semantics in the management of access control of these systems. Standards already exist for representing information in the health sector; we will represent these standards in OWL and use them as our policy subjects and resources. The standard for information representation in the health sector is Health Level 7 (HL7) [8]. Figure 4 shows the core classes of the HL7 Reference Information Model (RIM). These six core classes will be the principle classes in the ontology, with numerous subclasses extending from them.

Bhavna Orgun [9] has developed a similar ontology using Protégé [7]. We will further this by representing HL7 in OWL. 


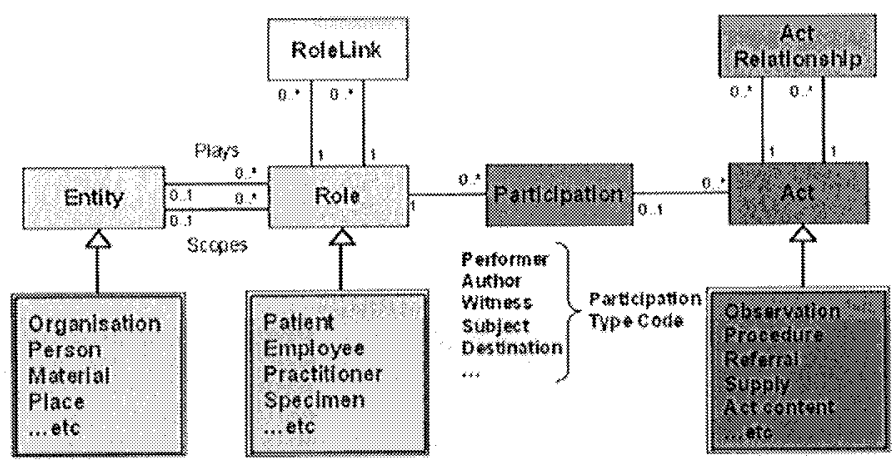

Figure 4. RIM Core Classes and Specialisations

The subjects and resources in our architecture are modelled as two separate items of storage. For the purpose of this case study, we will be representing all the information needed for policy reasoning in one ontology, as specified by HL7. Examples of rules which will need to be represented in our policy language are:

"Clinical information may only be accessed by clinical staff "Nurses may only access information on a patient under their care".

From a series of rules similar to this, our system will be able to determine, for example, if a particular nurse has access to the lab results for a particular patient.

\section{Related Research}

KAoS uses OWL for reasoning about policies. KAoS exploits ontologies for representing domain information describing organisations of people, agents and other computational actors. KAoS was initially designed as a policy language for complex software agents, but it is now being adapted to grid computing and Web Service environments. "Within the KAoS Policy Ontologies (KPO), a policy is an instance of the appropriate policy type (positive or negative authorization; positive or negative obligation) that defines the associated values for its properties" [10]. The KPO defines basic ontologies for actors, actions, groups, places and policies. KPOs are used for the analysis and inference of policies. The KAoS framework provides KPAT (KAoS Policy Administration Tool) for policy specification, modification and execution. While policies can be defined using KPAT, KAoS represents these policies in OWL. The user does not have to deal with policies at this level. KAoS can detect policy conflicts when they are being specified, using Stanfords Java Theorem 
Prover (JTP) [11]. KAoS will try and resolve this conflict by placing an order on the policies.

Rei is a distributed policy language that enables every Web entity to specify policies for its access, for privacy, for entities it wants to communicate with, etc., which are enforced either by an internal policy engine or the policy engine on the platform on which it is running or with which it is registered [12]. Rei v2 is written using OWL-Lite. Rei however extends OWL-Lite to include logiclike variables. Policies defined in Rei are described in terms of [13]:

- permissions - able to do something

- prohibitions - not able to do something

- obligations - should do something

- dispensations - should not do something

These are grouped into permissibility and obligation. These four terms are known as deontic objects as defined in deontic logic. The Rei framework provides a policy engine that reasons about the policy specification. Upon loading, the Rei engine will detect any potential conflicts within policies.

Qin et al propose "an access control model for the Semantic Web that is capable of specifying authorizations over concepts defined in ontologies and enforcing them upon data instances annotated by the concepts" [14]. To this end, Qin et al propose a solution that not only grants access to a subject, on an object at an element, document and DTD level, but also at a concept level. The novel approach to access control by proposed by Qin er al is not limited to access restriction at a concept level; it also proposes the ability to propagate these access policies based on the semantic relationships among concepts or ontologies. They present an OWL-based access control language SACL (Semantic Access Control Language) as the language used to create authorisation policies in their proposed model. SACL is an extension of OWL. It has such additions as SACL:higherLevelThan and SACL:lowerLevelThan, to specify ordering, and 'canRead' and 'readBy' to specify privileges.

Parsia et al, in [15], propose a semantically-aware policy language by translating WS-Policy [16] into OWL-DL. They propose two translations; the first translates policies into OWL-DL classes. In the first case the WS-Policy grammar is encoded in OWL, whereas in the second case the actual formalism underlying the WS-Policy grammar is captured in OWL. To represent policies in OWL instances, Parsia et al define two particular OWL classes, one to represent WS-Policy assertions and the other to represent WS-Policy alternatives. Policy assertions usually deal with domain specific knowledge. Alternatives are groups of assertions, each of which must be satisfied by the requestor for the alternative to be satisfied. The second case "maps the WS-Policy fromalism directly in OWL" [15]. First of all policy assertions are mapped into OWL-DL 
atomic classes. Since assertions are now classes, relationships between these classes must be defined.

Damiani et al [17] outline how "current standard policy languages such as XACML can be extended" to be able to semantically define access control policies for the Semantic Web. They propose the use of RDF to make the XACML policies more semantically aware. They extend XACML to include data describing subjects and resources, to use RDF assertions or user defined properties and to define some policy processing information. Damiani et al's policy evaluation engine performs two principle activities; the comparison of the user assertions in the request and the user descriptions in the ontologies to identify appropriate policy rules; and the querying of resource descriptions to determine if the requesting user satisfies these rules.

\section{Conclusions}

Existing standards for access control are quite restrictive. Recent advancements in XACML have provided generic attributes of a requestor and resources but do not harness the expressive power and reasoning capabilities of the Semantic Web. We have presented a proposed access control model which we believe has selected the best attributes of other solutions in the area. We can see from the previous section that new ontologies in the Semantic Web, especially those written for the Web, are being built using one of the OWL languages. It is quite important to follow the trends of the community, in our opinion. We have therefore selected OWL as the language with which we will create our knowledge-bases.

The importance of standards cannot be emphasised strongly enough. Within the realm of Web Services security, XACML has become the front runner in defining access control. It is important for us to use this architecture, as agreed by W3C [18], as the basis for our proposed solution. By coupling XACML with OWL access control rules can be represented in a standard logic and can therefore benefit from the tools and expertise associated with popular standards such as these.

From the solutions described in Section 5 Rei, KAoS and the idea proposed by Parsia et al [15] are more concerned with policies that can be exchanged between communicating parties and how they can be enforced. This is certainly the nature of WS-Policy in the Web Services Security arena. We propose using $\mathrm{XACML}$ which is a standard for representing access control rules. Although exportable policies can be deduced from these rules, it is not the principal goal. Damiani et al [17] also base their solution on XACML yet represent the rules in RDF. OWL-DL yields maximum expressiveness without loosing computational completeness. 
Acknowledgements. This work is funded by Enterprise Ireland as part of the Advanced Technology Research Program.

\section{References}

[1] Gray, Matthew. "Internet Growth Summary". Available at "http://www.w3.org".

[2] eXtensible Access Control Markup Language (XACML). Available at "http://docs.oasisopen.org/xacml/access_control-xacml-2_0-core-spec-cd-04.pdf".

[3] O’Neill M.Web Services Security, McGraw-Hill/Osborne, 2003.

[4] OWL Web Ontology Language. Available at "http://www.w3.org/TR/owl-features/".

[5] Klien, M. "Beyond RDF Schema". Available at "http://dit.unitn.it/ bouquet/kr-2005/slideslibro/wbkr_c6.pdf".

[6] XML Key Managemant Specification (XKMS), Available at "http://www.w3.org/TR/xkms/".

[7] Protégé. Available at "http://protege.stanford.edu/".

[8] Health Level 7 version 3. Available at "http://www.hl7.org/".

[9] Orgun, B. "http://www.ics.mq.edu.au/ borgun".

[10] Tonti, G., J. M. Bradshaw, R. Jeffers, R. Montanari, N. Suri and A. Uszok. "Semantic Web Languages for Policy Representation and Reasoning: A Conparison of KAoS, Rei, and Ponder". In Proceedings of 2nd International Semantic Web Conference,Sanibel Islands, Florida, USA. 2003.

[11] JTP-Java Theorm Prover. Available at "http://www.ksl.stanford.edu/software/JTP/".

[12] Kagal, L., T. Finin and A. Joshi. "A Policy Based Approach to Security for the Semantic Web". In Proceedings of 2nd International Semantic Web Conference, Sanibel Islands, Florida, USA. 2003.

[13] Kagal, L. "A Policy Based Approach to Governing Autonomous Behaviour in Distributed Environments". PhD Dissertation, University of Maryland, Baltimore, MD, USA. 2004.

[14] Qin, L. and V. Atluri. "Concept-Level Access Control for the Semantic Web". In proceedings of ACM Workshop on XML Security, Fairfax, VA, USA. 2003.

[15] Parsia, B., V. Kolozski and J. Hendler. "Expressing WS Policies in OWL". In proceedings of 14th International World Wide Web Conference, Chiba, Japan. 2005.

[16] Web Services Policy Framework (WS-Policy). Available at "ftp://www6.software.ibm.com/software/developer/library/ws-policy.pdf".

[17] Damiani, E., S. De Capatani di Vimercati, C. Fugazza and P. Samarati. "Extending Policy Languages to the Semantic Web". In proceedings of 4th International Conference on Web Engineering, Munich, Germany. 2004.

[18] World Wide Web Consortium (W3C), "http://www.w3.org". 